\title{
Feministas en sus zapatos: historiografía y enseñanza de la Historia ${ }^{1}$
}

por Cecilia Maria Rugna Cuenca

Universidad Nacional del Litoral / IDAES-Universidad de San Martín cecilia_rugna@hotmail.com

Recibido: 21|04|2014 · Aceptado: 23|05|2014

\section{Resumen}

En este artículo transito un recorrido que vincula Historiografía e Historia enseñada. Considero a la Historiografía que me enseñaron y aprendí en las aulas de la Universidad, desde la teoría y praxis de los feminismos, como productora del «plano inclinado». Esta metáfora de Margarita Pisano ilustra la situación de exclusión y silenciamiento del colectivo mujeres con respecto a la construcción del saber y a la forma de estar con/en el saber. La ejemplificación y justifi

tiones: a) los aspectos problemáticos y escurridizos de la Ley No 26150 de Educación Sexual Integral en el momento en que promueve la transversalización de contenidos con perspectiva de género a quienes han estudiado versiones tradicionales de la Historiografía; b) las estrategias (herramientas conceptuales, metodológicas, etc.) que pueden implementarse para revertir esa situación, principalmente teniendo en cuenta los aportes que surgen de la distinción entre Historia contributiva y crítica.

Palabras clave

Historiografía, Historia enseñada, feminismo, educación sexual integral, Historia crítica.

ers

\section{Feminists in their shoes: historiography and history teaching}

\section{Abstract}

In this article I transit a route linking Historiography and History taught. I consider historiography taught me and I learned in the classrooms of the University, from the theory and praxis of feminism, as producer of the «inclined plane». Th illustrates exclusion and silencing of women regarding collective construction of knowledge and the way of being with / in the know. The modeling and justification of the existence of the «inclined plane» is key to investigate two questions: a) the problematic and elusive aspects of the sexual education's law in the moment content promotes mainstreaming a gender perspec- 
tive to those who have studied traditional versions of Historiography; b) strategies (conceptual, methodological, etc..) that can be implemented to reverse this situation, mainly taking into account the contributions arising from the distinction between contributory History and critical History.

Keywords

Historiography, History taught, feminism, sexual education, critical History.

En este trabajo me propongo refl

la Historiografía que me enseñaron y aprendí en las aulas de la Universidad reconociéndola como parte del «plano inclinado» que la pensadora feminista Margarita Pisano describe. Ese reconocimiento del plano inclinado resultará clave para indagar cuáles son los contenidos que aprendemos y sus límites en los diseños curriculares; qué y cómo enseñamos, cómo son recibidas las nuevas propuestas que proponen contenidos distintos a los que nos fueron legados en nuestra formación, etc. Para este fi

tanto la investigación histórica como la educación en Historia.

Dividiré el artículo en secciones, útiles para ordenar la exposición de ideas. En primer lugar, analizaré cómo se expresa en hechos concretos de la Historiografía el plano inclinado al cual hace referencia Margarita Pisano. En segundo lugar, y relacionado con lo anterior, problematizaré la noción de transversalización de contenidos que plantea la Ley No 26150, de creación del Programa de Educación Sexual Integral. Por transversalización se entiende que lxs docentes deben incluir la perspectiva de género en los contenidos que figuran en la currícula de Historia. Mi pregunta es básicamente: ¿cómo enseñar lo que no he aprendido formalmente en el ámbito escolar? ¿Cómo se transversaliza si no se estudia en profundidad el concepto de género y su Historia? ¿Cómo si se desconoce la biblioteca feminista? (la biblioteca feminista introduce otros temas al irrumpir en lo que comúnmente se entiende por sexo, sexualidad, trabajo, familia, reproducción, producción, tiempo, etcétera).

En tercer lugar, nos centraremos en la historiografía que podríamos producir y enseñar desde los saberes que los feminismos aportan para construir otros contenidos en Historia diferentes a los tradicionales - y que sí contemplen la perspectiva de género. La mención a la Historia tradicional puede ser leída aquí como una provocación, ya que, se suele denominar como tradicional a las escuelas decimonónicas, que recalaban sumariamente en los "grandes» personajes y en la política; y no precisamente a la contemporánea de las aulas universitarias. Sin embargo, seguir privilegiando aspectos económicos y políticos para hacer Historia y reducir la llamada Historia Social a cuestiones propias y exclusivamente masculinistas, denota poca vocación de transformación.

Sugeriré estrategias para revertir ese plano inclinado, esto es, que haya o tenga lugar la Historiografía feminista desde la certeza que mirando lo realizado podremos construir el diagnóstico para entender por qué las mujeres están invisibilizadas en el discurso historiográfico. 